\title{
Model-Based Integration Platform for FMI Co-Simulation and Heter- ogeneous Simulations of Cyber-Physical Systems
}

\author{
Himanshu Neema ${ }^{1}$ \\ Janos Sztipanovits ${ }^{1}$ \\ John Batteh ${ }^{2}$

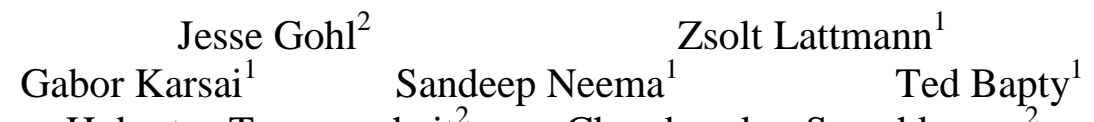 \\ Gabor Karsai $^{\text {Jesse Gohl }^{2}}$ Sandeep Neema $^{{ }^{1}}$ Zsolt Lattmann $^{1}$ Ted Bapty $^{1}$ \\ ${ }^{1}$ Institute for Software Integrated Systems, Vanderbilt University \\ 1025 16th Avenue South, Suite 102, Nashville, TN 37212, USA \\ ${ }^{2}$ Modelon, Inc. \\ 2389 Main Street, Glastonbury, CT 06033, USA \\ himanshu@isis.vanderbilt.edu jesse.gohl@modelon.com lattmann@isis.vanderbilt.edu szt- \\ ipaj@isis.vanderbilt.edu gabor@isis.vanderbilt.edu sandeep@isis.vanderbilt.edu bap- \\ ty@isis.vanderbilt.edu john.batteh@modelon.com hubertus.tummescheit@modelon.com chandra- \\ sekar.sureshkumar@modelon.com
}

\begin{abstract}
Virtual evaluation of complex Cyber-Physical Systems (CPS) [1] with a number of tightly integrated domains such as physical, mechanical, electrical, thermal, cyber, etc. demand the use of heterogeneous simulation environments. Our previous effort with C2 Wind Tunnel (C2WT) [2] [3] attempted to solve the challenges of evaluating these complex systems as-a-whole, by integrating multiple simulation platforms with varying semantics and integrating and managing different simulation models and their interactions. Recently, a great interest has developed to use Functional Mockup Interface (FMI) [4] for a variety of dynamics simulation packages, particularly in the automotive industry. Leveraging the C2WT effort on effective integration of different simulation engines with different Models of Computation (MoCs), we propose, in this paper, to use the proven methods of High-Level Architecture (HLA)-based model and system integration. We identify the challenges of integrating Functional Mockup Unit for Co-Simulation (FMU-CS) in general and via HLA [5] and present a novel model-based approach to rapidly synthesize an effective integration. The approach presented provides a unique opportunity to integrate readily available FMU-CS components with various specialized simulation packages to rapidly synthesize HLA-based integrated simulations for the overall composed Cyber-Physical Systems.
\end{abstract}

Keywords: Functional Mockup Interface, Functional Mock-up Unit for Co-Simulation, Cyber-Physical Systems, Heterogeneous simulation, Multi-paradigm modeling, Model-based integration, DSML, Distributed Simulation, High-Level Architecture

\section{Introduction}

Cyber-Physical Systems (CPS) [1] are composed of several collaborating physical and computing components that interact through embedded communication capabilities. These systems require advanced integration of abstractions and techniques that have been developed over the past years in disparate areas such as cyber systems that rely heavily on computation and networking and physical systems that employ various engineering methods in domains such as mechanical, thermal, electrical, electronic, hydraulic, thermal, biological, and acoustic.

Analysis of Cyber-Physical Systems poses unique challenges due to the heterogeneity of components and interactions [2]. The fundamental differences in the characteristics of these different physical and computation processes lead to a huge spectrum of modeling methods. For example, some components can be easily described by differential equations, while others like communication networks are better modeled as Discrete-Event Systems. As such, several simulation tools and techniques are needed for CPS simulation and analysis. This further necessitates an over-arching CPS model and system integration platform that is model-based and supports rapid synthesis of distributed heterogeneous CPS simulations.

Co-Simulation (Co-operative Simulation) is a simulation method that permits simulating individual components using different simulation tools simulta- 
neously and collaboratively. Individual simulation tools exchange information such as system variables and their values, time steps for synchronization, and control signals for orchestrating the co-operative simulation. Thus, engineers can use different simulation tools together to create virtual prototypes of entire Cyber-Physical Systems. In practice, however, significant challenges remain with regard to the syntax and semantics of model and system integration.

In the Co-Simulation domain, a recent effort by the MODELISAR ITEA2 project that develops a tool independent standard called the Functional Mock-up Interface (FMI) [4] [7] [8] has gained significant influence, more prominently in the automotive industry. The FMI standard provides a welldefined specification and API to integrate simulation components. FMI-compliant simulations pack shared libraries that can be executed using the standardized function calls and the model execution must adhere to the rules of the standard. These function calls span all stages of the model execution, viz. initialization, configuration, access, modification, and manipulation.

The strength of FMI lies in the fact that all simulation tools participating in the Co-Simulation follow the defined standard and as such provides for standardized access to model equations. This permits coupling of Continuous-Time, Discrete-Time, and Discrete-Event that are part of a Cyber-Physical System. In some ways, this is also a limitation because not all simulation tools are amenable to support all of the strictly specified FMI function calls.

Another key requirement for Co-Simulation via FMI is to also develop a Master Algorithm (MA) that orchestrates the steps of Co-Simulation. Master algorithms have two tasks: (1) control the data exchange and (2) control time advancement among individual simulations according to the requirements of the integrated simulation of the overall CyberPhysical System. Since the FMI standard does not describe or limit the implementation of the MAs, it basically leaves out the two fundamental challenges that all distributed simulation architectures face: data exchange and time management ${ }^{l}$. Solution for integrated data and time management in distributed simulations is technically complex and errors can easily lead to performance bottleneck and failures. This complexity pushes designers to adhere to the simplest solutions - losing much of the potential advantages of co-simulation.

\footnotetext{
1 Theoretically, an MA for direct co-ordination among FMUs can be developed for each distributed simulation problem, but that would be expensive and subject to errors.
}

For example, Cyber-Physical Systems frequently involve vastly different sub-domains and physical processes that vary greatly in the execution frequency at which they need to run. This leads to significantly different dynamic response characteristics. For example, mechanical components of a complex CPS often have much slower frequency responses compared to fast electronic components. A single standalone monolithic model of a CPS therefore suffers heavily with solver inefficiencies. These systems are generally highly complex and have significant non-linearities and discontinuities, which further increases difficulties of gaining convergent solutions. Taking subsystems apart and using different solvers and step-sizes offers a potential solution. However, multirate composition also introduces inefficiencies due to time management, composition restrictions, data exchange, and potential stability issues if the system is split at the wrong place.

Challenges of distributed simulation have a long research history with notable results and standards. One of the widely used solutions developed by the U.S. Modeling and Simulation Coordination Office (M\&S CO) is the High Level Architecture (HLA) [5]. The HLA provides a specification of a common technical architecture for modeling and simulation with a primary goal to facilitate interoperability among simulations and to promote re-use of simulations and their components. The HLA comprises of three major components: HLA rules, HLA interface specification, and HLA object model template [5]. With these rules, the HLA standardizes run-time support for various tasks, such as coordinated time evolution, message passing and shared object management. The key difference between FMI and HLA is that HLA provides a standard for data exchange and time management for individual simulation processes. This enables broader integration of different simulation tools using different Models of Computation. Functional Mock-up Units can be integrated as a participating simulation tool in the overall integrated simulation of the Cyber-Physical Systems.

A key benefit of HLA is that its Distributed Discrete Event model of computation allows full flexibility to individual subsystems in using any internal solver and model of computation. Moreover, this flexibility permits multirate simulations by design.

However, the HLA standard also lacks some key facilities for developing integrated distributed heterogeneous simulations. For example, the HLA standard does not formalize methods for developing interactions and objects used by HLA federates and it does not provide facilities for easily moving simulations from one computational node to other. Consequently, HLA-based simulations also require a sig- 
nificant amount of tedious and error-prone handdeveloped integration code.

Achieving the integrated simulation of CyberPhysical Systems require effective integration of a huge spectrum of models of physical processes, communication systems, exchanged information, and control mechanisms. As detailed above, the approaches of FMI and HLA both have their advantages and some key limitations. The approach of using HLA as a master algorithm enables use of FMUs in a Co-Simulation environment while also providing flexibility of using other types of nonFMU simulations [9]. The resulting framework can provide a much broader scale of simulation tools that can be used in the integrated simulation of CyberPhysical Systems. However, several gaps need to be filled in order to develop a platform that enables this integration in an efficient manner. A single efficient model-based platform is needed that:

- Enables modeling of interactions and shared objects between simulation tools

- Enables modeling and integration of systems with their data exchange mechanisms

- Enables modeling of deployment of simulation tools on distributed computational infrastructure

- Provides a standard master algorithm for FMI Co-Simulation

- Enables multirate modeling with dynamic time management

- Provides a set of tools to generate necessary artifacts for rapid synthesis of simulations

This paper attempts to address these important challenges in creating a single coherent platform for developing integrated distributed simulations of Cyber-Physical Systems. We build upon our previous work on a model-based integration platform called the Command and Control Wind Tunnel (C2WT) [2] [3].

The rest of the paper is organized as follows. Section 2 and 3 give an overview of the $\mathrm{C} 2$ Wind Tunnel and FMI for Co-Simulation respectively. We present our detailed model-based integration approach in Section 4 and provide a detailed case study with experimental results in Section 5. Section 6 provides the related work and Section 7 concludes the paper.

\section{C2 Wind Tunnel}

Over the past several years, we have developed a model-based multi-model integration platform called the Command and Control Wind Tunnel (C2WT) [2] [3]. It is an integrated, graphical, multi-model, dis- tributed simulation environment for the experimental evaluation of large-scale C2 systems with various organizational and technical architectures. It enables a variety of simulation engines to interact and transmit data from one another and log and analyze simulation results. Figure 1 below gives a conceptual architecture of C2WT.

The High-Level Architecture is a standardized framework for distributed computer simulation systems. Communications between different federates is managed via the Run-Time Infrastructure (RTI) layer. The RTI provides a set of services such as time management, data distribution, message passing, and ownership management. Other components of the HLA standard are the Object Model Template (OMT) and the Federate Interface Specification (FIS).

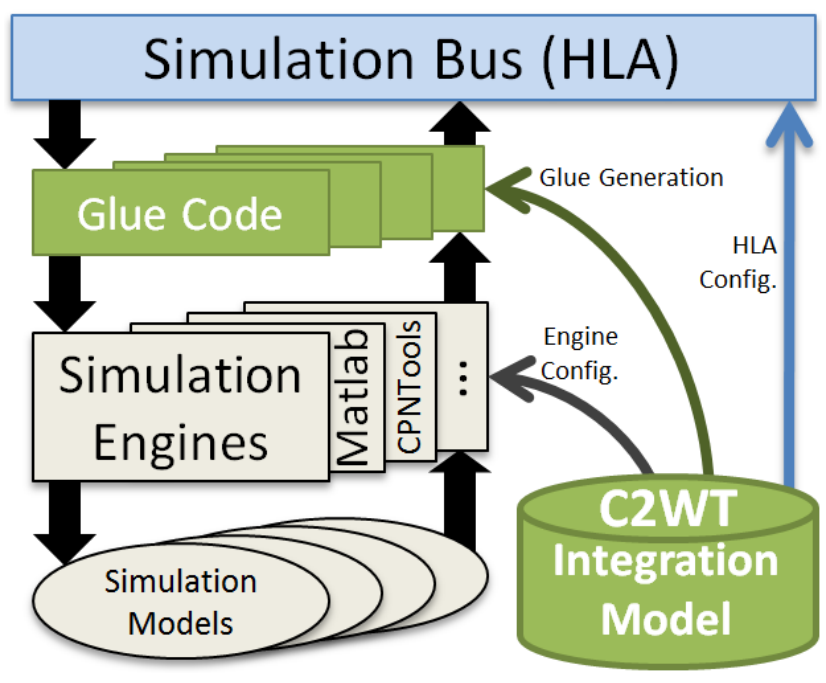

Figure 1: Conceptual architecture of C2WT

The HLA standard focuses on three primary areas. First is time coordination throughout the federation. The evolution of time is a key thread through each of the integrated simulators. Each simulation platform must slave its progression of time to that of the overall HLA clock. The HLA standard provides several methods by which to accomplish this. Second is coordination of inter-federate messages and shared data objects. The HLA standard provides a publish-and-subscribe mechanism for passing messages and object updates throughout the federation. Third, the HLA standard provides for basic simulation execution control. Starting, pausing, and stopping the execution of a simulation is built directly into the HLA standard. The $\mathrm{C} 2 \mathrm{~W}$ Wind Tunnel relies upon all of these services during run-time.

As HLA is an accepted standard, a number of commercial, academic, and alternate RTI implementations are available. Currently, we use the Portico 
RTI [10] - which provides support for both $\mathrm{C}++$ and Java clients and is compliant with version 1.3 of the HLA standard.

The HLA provides a standard for the RTI that supports the coordinated execution of distributed simulations. However, designing the model integration, coding the platform-to-RTI glue-code, and testing and deploying all of the various run-time components across multiple platform-specific simulation tools is a highly challenging task. C2WT provides a solution to this simulation integration problem. It provides a holistic modeling and management environment built around a custom Domain-Specific Modeling Language (DSML) [11], implemented in Generic Modeling Environment (GME) [11], and a related suite of model interpreters to coordinate between the integration model and the platformspecific simulation tools involved in the overall environment. It facilitates the rapid development of integration models and use of these models throughout the lifecycle of the simulated environment. With simulation engine specific model configurations and experiment specific deployment modeling, it enables significant automation in the development of integrated distributed simulation. With integration modeling support and various sophisticated generation tools, C2WT provides a robust platform for users to rapidly model and synthesize complex, heterogeneous, command and control simulations.

\section{FMI for Co-Simulation}

Functional Mock-up Interface (FMI) [4] [7] [8] was initiated and organized by Daimler AG within the ITEA2 project MODELISAR [4]. The FMI standard consists of two main parts. The first part is FMI for Model Exchange, which standardizes the distribution of a dynamic system model in the form of generated C-Code as an input/output block to other simulation environments. The second part is FMI for Co-Simulation, which standardizes the mechanisms for coupling of two or more simulation tools in a co-simulation environment.

The key idea is to have a discrete set of communication points only, at which times the subsystems exchange any data. Outside of these points, the subsystems are executed independently. The data exchange is controlled by a master system that also manages time synchronization of subsystems.

The FMI Co-simulation master simulator couples the subsystem simulators through a zip-archive. This zip-archive contains shared library files (.DLL, .SO) that conform to the function call specifications given in the standard. Each zip-archive also contains a
XML file that provides meta-data and further details of the model such as default start and stop times, variable types, units, tool specific data, parameter and variable names and attributes. The XML also contains specification for executing the model as a shared library during a simulation run (CoSimulation_Standalone) or by importing a slave tool wrapper and interfacing it with the external tool (CoSimulation_Tool).

\section{Model-Based Integration}

One of the primary contributions of our effort is our focus on developing a completely model-based integration approach. Our efforts leverage the Generic Modeling Environment (GME) [11] tool suite for designing the integration model DSML [11] and HLA [5] to provide run-time support as the "simulation bus".

\subsection{Needs and Challenges}

Cyber-Physical Systems [1] [2] are highly complex and their simulation spans a multitude of computational domains and specializations. A large number of tools exist that have been developed for specific aspects of CPSs. A variety of tools exist even for a single aspect of CPSs. For example, many special purpose simulation tools exist to model and analyze vehicle dynamics or for switching mechanisms of hybrid drivetrains. As such the integration platform must be open toward use of any tool that may be required for some component/aspect of the CPS simulation.

A subtle problem in using multiple simulation tools in an integrated simulation is that they tend to use many different Models of Computation (MoC). For example, Discrete-Event, Discrete-Time, Continuous-Time, Synchronous Dataflow, are among the many MoCs used. Each MoC has a specific mechanism for time progression and event handling. The integration platform must be able to handle tools that use different MoCs in highly flexible manner. The integrated system must respect time synchronization with other simulation tools as well as the causality of events must be preserved. In addition to system integration, the platform must also enable integration of models by means of capturing the communication (with any translation that might be needed) that occurs between them.

In general, it is preferable to have a graphical environment that provides well-defined semantics for modeling concepts, their relations, and rules for composition. Moreover, for rapid synthesis of simu- 
lations, the platform must support tools for translation of models to executable software that conform to specified executable semantics. The automation not only provides efficient development of simulations, it also significantly minimizes human errors.

The integration environment should also provide capabilities for modeling and configuration experimentation and logging.

Furthermore, when FMUs are integrated the rules of FMI must still be adhered to. Particularly, the models in the FMUs must be accessed, controlled, and manipulated using the function calls specified in the FMI standard.

\subsection{Meta-modeling}

The Generic Modeling Environment is a metaprogrammable model-integrated computing (MIC) [11] toolkit that supports the creation of rich domainspecific modeling and program synthesis environments. Configuration is accomplished through meta models, expressed as UML class diagrams, specifying the modeling paradigm of the application domain. Meta models characterize the abstract syntax of the domain-specific modeling language, defining which objects (i.e. boxes, connections, and attributes) are permissible in the language. Another way to envision this is that a DSML [11] is a schema or data model for all the possible models that can be expressed by a language. Using finite state machines as an example, the DSML would consist of states and transitions. From these elements any state machine can be realized. The inherent flexibility and extensibility of the GME [11] via meta models make it an ideal foundation for the $\mathrm{C} 2$ Wind Tunnel environment. Alternate meta modeling frameworks have also been developed in the past, such as AToM3 [12], MetaCase [13], Microsoft DSL [14], and the Eclipse Modeling Framework [15].

\subsection{Model-Based Integration of FMUs in C2WT}

As detailed in section 2, C2WT provides an overarching modeling and management environment and a suite of model interpreters to coordinate the integration models and platform-specific simulation tools involved in the overall heterogeneous distributed simulations. The user is referred to [2] for details of the meta-modeling language and its executable semantics. In this section, we further discuss the integration of FMUs as HLA-federates in the C2WT platform.

In this work, the C2WT metamodel was further customized to enable FMU specific federate specifi- cations. Although the original C2WT metamodel is sufficient to support integration of newer types of federates, having simulation tool/technique specific first-class objects in the modeling language makes reasoning about such entities more flexible and can support extensive automation. The FMU-federate model specifies the location of the zip archive, whether to $\log$ variable values during simulation, additional variables (other than input and output) to $\log$, and ratio of macro and micro steps for multirate simulations.

Figure 2 below shows the extension to the original C2WT architecture to incorporate FMU federates in the platform.

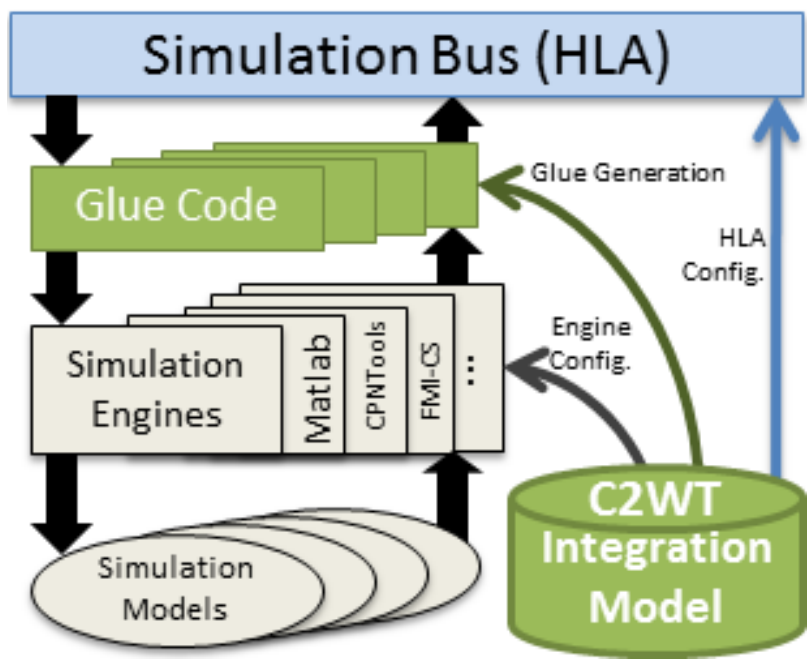

Figure 2: C2WT extended for FMI-CS

Our model interpreters can read the models with specified input and output relationships with other simulation tools and even other FMUs and can automatically generate all the executable code that can be deployed on different nodes in the available computational infrastructure for the simulation. As previously mentioned, C2WT supports simple modeling of computational infrastructure and assignment of federates on its nodes.

Following the rules of FMU access, modification, and manipulation as described in the FMI standard [4] [7], we developed a simplified procedure for FMU-federate execution as given below:

\section{Initialization phase (before simulation start):}

1: Load FMU zip archive, read model description

2: Load shared libraries in the FMU

3: Instantiate the FMU slave

4: Setup input/output and HLA-interaction maps

5: Setup up logging 


\section{Execution phase (during simulation):}

1: Synchronize start of simulation with all tools

2: Request RTI to proceed to step-size and wait

3: Update input variables with HLA updates

4: Call doStep in step-size/\#micro-steps chunks

5: Continue \#4 until full step-size is executed ${ }^{2}$.

6: Update HLA with output variables

7: Go to \#2

Please note that above is rather simplified procedure of FMU integration mechanism in C2WT. The actual implementation also involves setting up statistical and database logging, micro-step management to avoid overlaps, error-handling, efficient federate code execution, reliable \& reusable time advancing facilities, and model state and HLA interaction synchronization.

\section{Case Study}

To illustrate our model-based approach for FMU integration in C2WT we present a high-fidelity model of a representation of a Vehicle Thermal Management (VTM) system which is intended for studying interactions of thermal management systems within a vehicle.

\subsection{Model description}

This particular example is a conventional four wheel chassis and drivetrain architecture with a spark ignition engine and standard transmission. These mechanical systems are created using components from the Vehicle Dynamics Library (VDL) from Modelon [16]. The model also includes a representation of the coolant loop for the engine and transmission oil loop in conjunction with a four heat exchanger stack for the thermal domain. These portions of the model are constructed from components of the Liquid Cooling Library (LCL) from Modelon. A snapshot of the overall model is shown in the following Figure 3 below.

The key component models of the system are: Driver, Vehicle (Engine, Transmission, Driveline, Chassis, Aerodynamics, External loads, and Brakes), Lumped engine thermal mass, Lumped transmission thermal mass, Engine coolant fluid circuit, Transmis-

\footnotetext{
${ }^{2}$ Note that an FMU freely updates its internal variables during micro-steps, but the input variables at the beginning of step \#4, remain unchanged during step \#5. Each subsequent micro-step, within the step \#5, uses the updated internal variables in previous micro-step. Also, it is assumed that FMUs do not reject time-steps.
}

sion oil cooling circuit, Heat exchanger stack, Low voltage battery, Alternator, Cooling fan and controller, and Grill shutters and controller. Table 1 below provides key features of these component models.

Since the purpose of this model is to study vehicle thermal dynamics, a simplified 1D longitudinal dynamics chassis model is used rather than a full 3D body model. This allows for faster simulations of the typically long duration drive cycles.

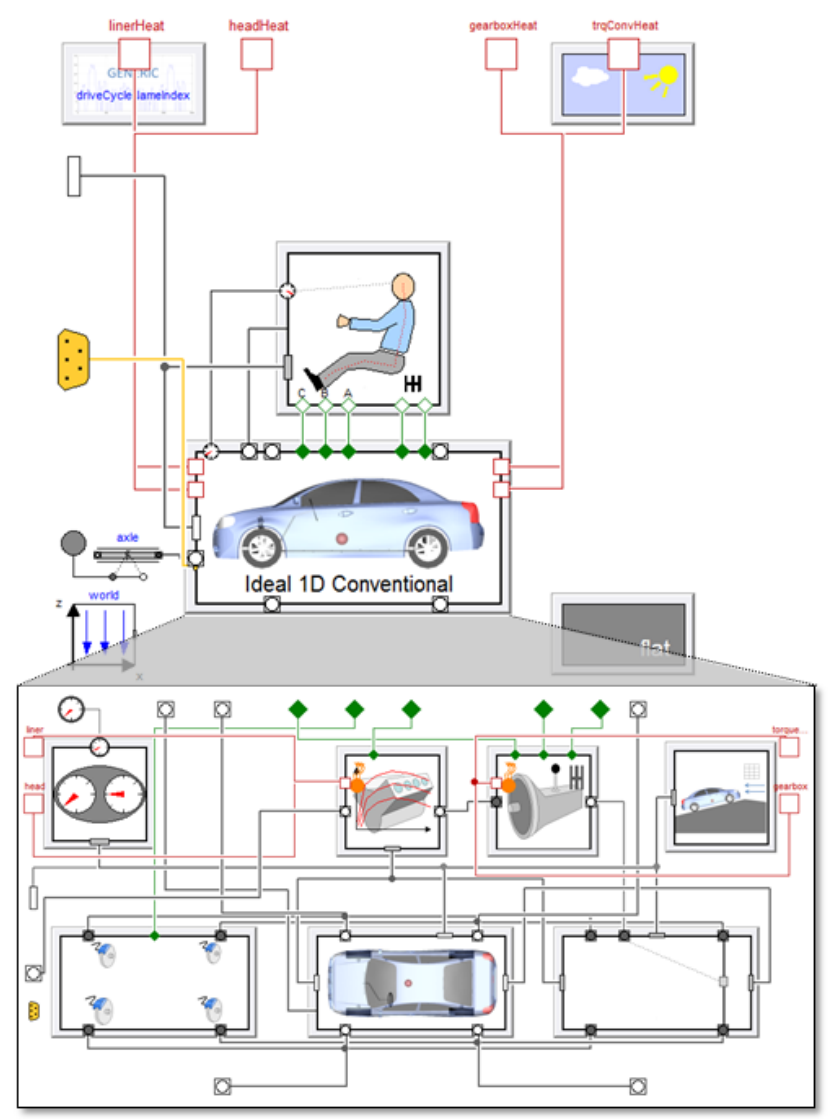

Figure 3: Overall system model

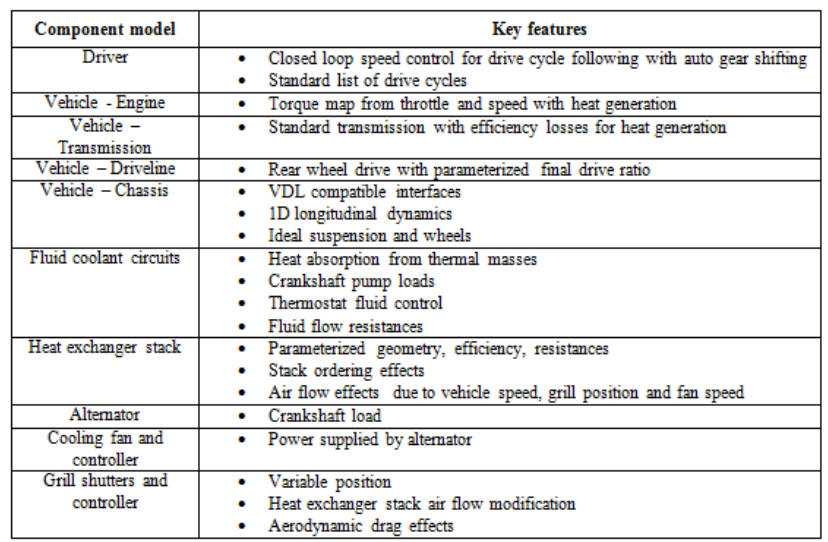

Table 1: Key features of component models 
During the simulation, heat that is generated by the engine is stored within the engine thermal mass and then rejected to the coolant-to-air heat exchanger (radiator) through a coolant fluid loop. A similar loop and heat exchanger also exists for the transmission.

The model is well suited to thermal management controller design, studying tradeoffs between thermal management energy demands and fuel economy, heat exchanger efficiency and sizing, and coolant fluid flow dynamics.

For this paper, the model was partitioned into separate executables by dividing the model along domain boundaries. In this case the vehicle mechanics, electrical, and driver were grouped into one model while the fluid and thermal portions of the model were grouped into another. This partitioning allows for execution of Driver vehicle and Thermal management parts at different rates. Owing to the inclusion of fluid portions in the Thermal management part, this part needed to run with a much lower step-size than the Driver vehicle part to maintain system stability.

In order to do this the physical connections that are bisected by the boundaries must be converted to causal signals. As an example for the engine, the heat is generated within the mechanical portion of the model. The heat is directed to the lumped thermal model, within the thermal portion of the model, which determines the thermal mass temperature. Images of these two systems are shown in Figures 4 and 5 below.

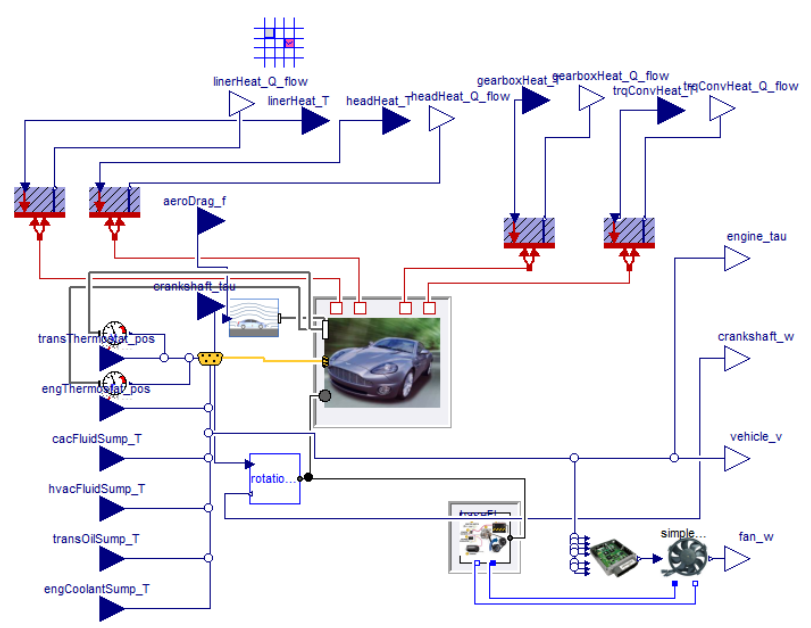

Figure 4: Driver vehicle model

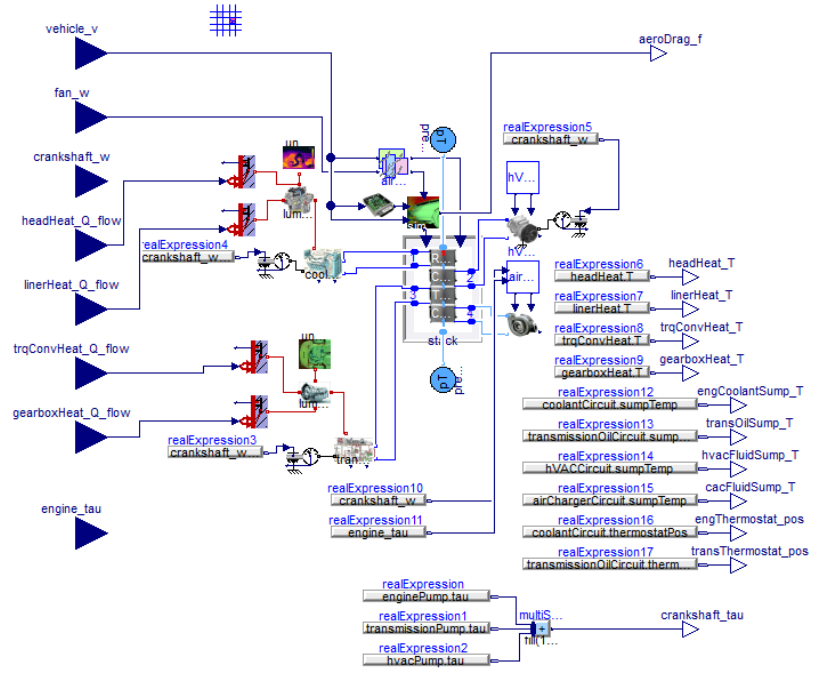

Figure 5: Thermal management model

\subsection{Simulation architecture}

The simulation setup consisted of mainly three federates, viz. Driver vehicle, Thermal management, and the Manager federate. Manager federate is an auto-generated external federate, which is used mainly as a front-end controller of the overall heterogeneous simulation. The simulation architecture is illustrated in the Figure 6 below.

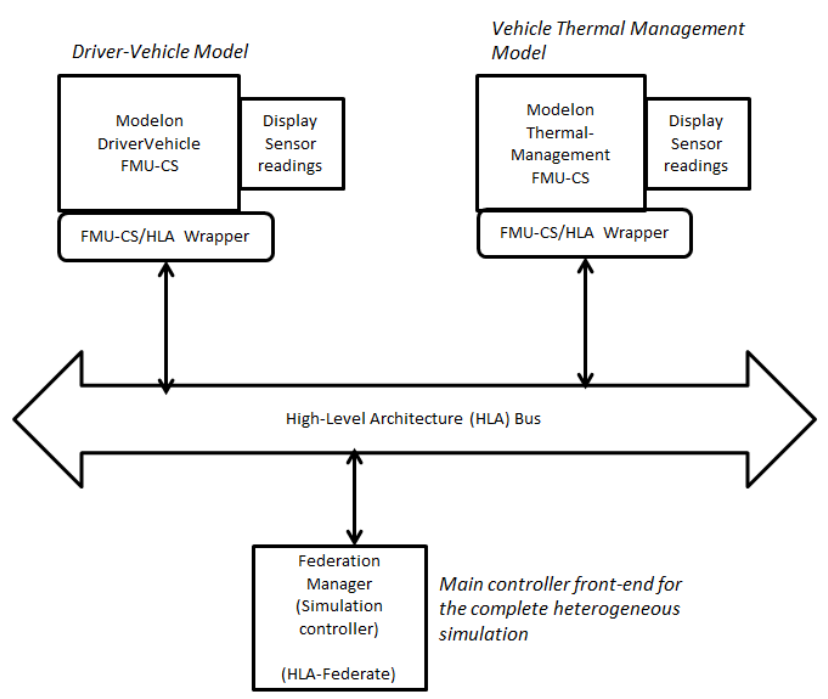

Figure 6: Simulation architecture

\subsection{Data and Integration model}

The actual data and integration model are given in the Figures 7 and 8 below. These show the input and variables from the Driver vehicle and Thermal management federates. These two models are executed as FMUs in the C2WT. 


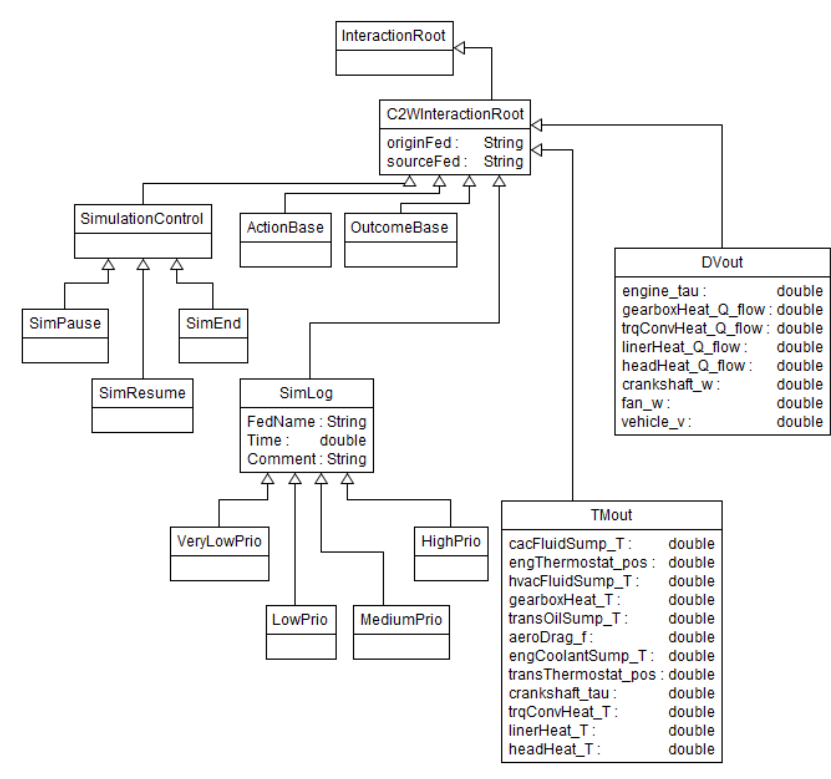

Figure 7: Data model

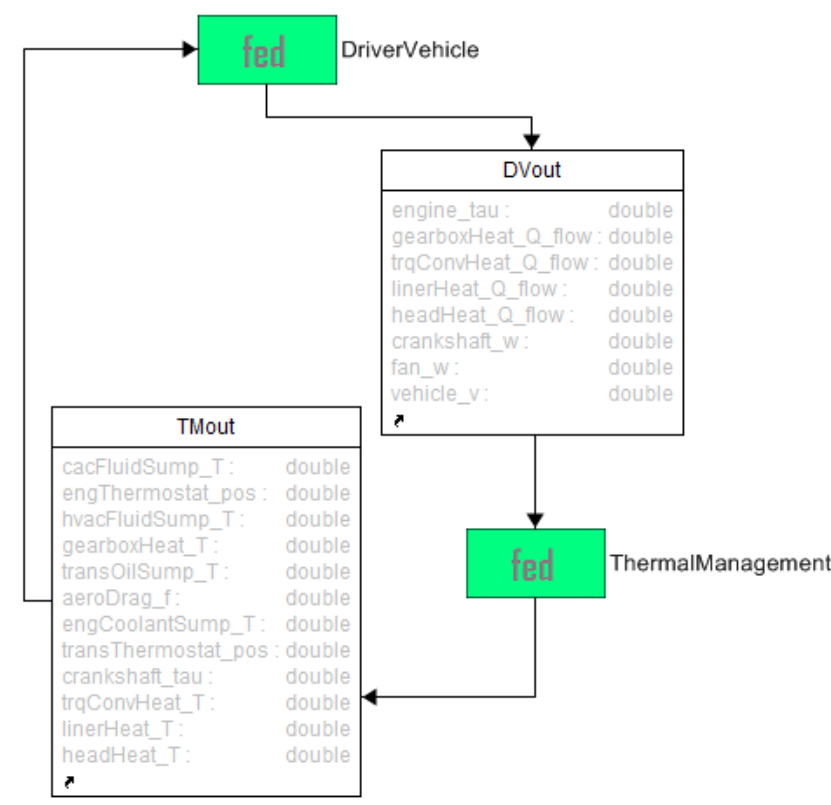

Figure 8: Integration model

\subsection{Experimental Results}

For the experiment, the Driver vehicle and Thermal management FMUs were exported from Dymola [16] models by Modelon, Inc. [16]. We used a JFMI Ptolemy APIs [17] to connect the FMUs to our Java based C2WT platform. All federates were running in a single Ubuntu 32 virtual machine. The Run-Time Infrastructure (RTI) used was Portico [10]. Total simulation time for the experiment was 50 seconds.
The simulation was setup as a multirate simulation with different step-sizes for the three federates: Driver vehicle $(10 \mathrm{~ms})$, Thermal management (5 ms), and Federation Manager (100 ms). The entire simulation ran in about $\sim 9$ minutes. The Figures 9 and 10 above show the experimental results for the total 50 seconds of simulation time. It should be noted though that the VTM models used were currently not optimized for efficiency.

From the experimental results, we found closely matching plots with same peak and trough values that were in the equivalent single monolithic (combined Driver vehicle and Thermal management) model. The overall runtime ( $\sim 9$ minutes) was also comparable to standalone single model simulation time in Dymola ( 6 minutes) despite the use of a third federate (viz. Manager federate) in the simulation and delays due to inter-process communications.

The models were developed with a variable step solver as requirement. However, they could still run with a fixed step solver (with a maximum step-size of $1.5 \mathrm{~ms}$ ). However, with our setup of separating the Driver vehicle and Thermal management components as separate FMUs and executing them through C2WT platform, we could even execute these components at $10 \mathrm{~ms}$ and $5 \mathrm{~ms}$ step-sizes respectively.
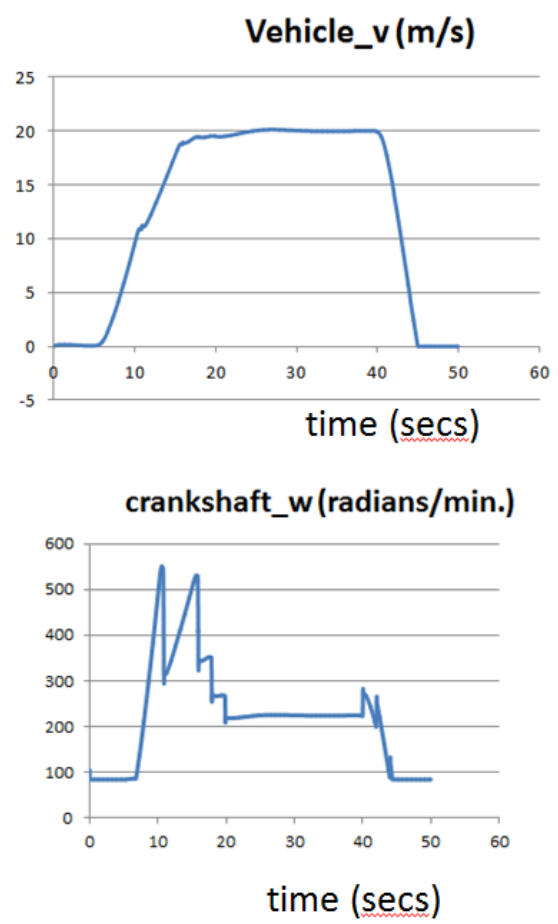

Figure 9: Vehicle speed and crankshaft angular velocity 


\section{ideal1DSedanCycleFollower.driver.ge} arRobot.gearSelection
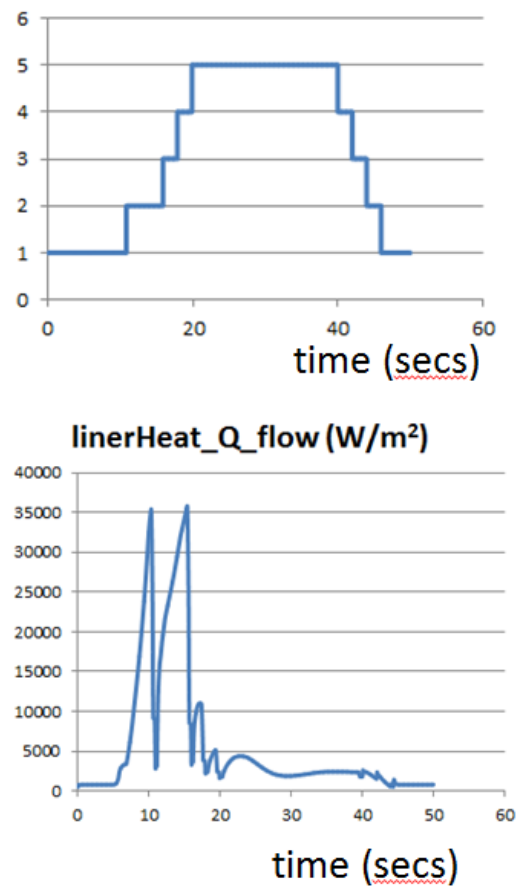

Figure 10: Gear selection and Liner heat flux

Yet another experiment we have performed is the one where we placed a network simulator for the CAN bus that must be placed between the above two components. We used the OMNeT++ simulator [18] to model that. In this experiment, we varied the rates of the FMUs to initially match the rate at which network simulator was run, viz. $0.5 \mathrm{~ms}$, and then in the second setup we increased the step-size of Driver vehicle and Thermal management to $1 \mathrm{~ms}$. We found that the results still matched while in the second setup they executed in about one-third the overall wallclock time. We omit further details of experiment setup here for brevity. In the future, we plan to further publish our work focusing specifically on the multirate aspects in distributed CPS simulations.

\section{Related Work}

Distributed simulation of Cyber-Physical Systems [1] is a highly challenging endeavor with its unique set of requirements. In the past a number of researchers have attempted to solve these problems.

Some approaches aim at providing an integration mechanism for two or three tools used in CPS simulation. For example, [20] shows integrated CPS simulation by coupling Matlab and a network simulation called EPANET, [21] presents an integration approach using SysML and numerical solvers, and [22] presents a co-simulation platform using Modelica framework and the ns- 2 network simulator. There are also approaches to provide a complete dedicated simulation environment. For example, [23] [24] presented a unified software environment for modelbased integration and multi-granular evaluation of widely used simulators. A simple master algorithm for Co-Simulation using FMI was given in [25] and a framework providing a central master simulator for FMUs as well as live components was provided in [26]. A dedicated environment for modeling with different models of computations and their execution via HLA was presented in [27]. Also, a cosimulation tool-chain has been proposed in [28], where existing system components from domains such as TinyOS and Modelica can be imported into SysML and then converted into corresponding FMU blocks. It was recently argued in [29] that it may be feasible to create a HLA-based master algorithm for FMI Co-simulations and then later a method was proposed in [9] [30] [31].

Our work focusses on providing a framework to enable model-based rapid synthesis on CPS simulations. The origins of our model-based approach can be found in [32]. Further, [33] explains how modelbased rapid synthesis can lead to development of Cyber-Physical Systems with continuous integration techniques. The C2WT framework [2] developed at our institute facilitates model-based rapid integration of heterogeneous and distributed simulations. Recently, C2WT framework was used to develop an evaluation tool for networked control systems in [34].

\section{Conclusions}

In this paper, we have successfully demonstrated a model-based integration approach to rapidly synthesize multi-model distributed simulation that may also involve co-simulation FMUs as component models. The FMUs are automatically wrapped as HLA-federates that can be executed in the C2WT platform.

We also illustrated that different federates can be run with different clocks and their synchronization in C2WT is managed using HLA time management facilities. We have also integrated FMU-CS in simulations that also use other simulation tools such as a network simulator or a 3D terrain simulator. The integration of other federates in C2WT has been previously demonstrated in [2]. Thus C2WT provides a broader range of simulation tool integration that involves FMI and non-FMI simulations to enable development of System-of-System (SOS) simulations. 
C2WT supports real-time and as-fast-as-possible modes of simulation execution. However, currently the real-time simulation requires that the individual component simulations can run faster than real-time.

C2WT also supports human-in-the-loop simulations with real-time simulations. In this case human interaction with running simulations (e.g. in military training exercises) is performed using HLAinteraction mappings.

One of the key benefits of C2WT platform is its support for extensive experimentation, message logging, state variables logging, and analysis support.

The research at our institute is currently ongoing with the applications of FMI Co-Simulation using HLA-based integrations. We anticipate novel methods for FMI Co-Simulations that are rapidly synthesized and may perform faster than single monolithic simulations.

We are also working on extending the C2WT platform to support other simulation techniques and tools such as SystemC.

\section{Acknowledgements}

The authors acknowledge financial support from the US DoD's Defense Advanced Research Projects Agency under the project "Adaptive Vehicle Make" [19]. We also acknowledge the invaluable contributions for the efforts in this paper from our collaborators at Modelon, Inc. [16].

\section{References}

[1] J. Sztipanovits, "Composition of cyberphysical systems," 14th Annual IEEE Int'l. Conference and Workshops on the Engineering of Computer-Based Systems (ECBS '07). Washington, DC, USA: IEEE Computer Society, 2007, pp. 3-6.

[2] Graham Hemingway, Himanshu Neema, Harmon Nine, Janos Sztipanovits, Gabor Karsai, "Rapid Synthesis of High-Level Architecture-Based Heterogeneous Simulation: A Model-Based Integration Approach", Simulation 88(2), 217-232 (2012)

[3] C2WT community wiki wiki.isis.vanderbilt.edu/OpenC2WT

[4] Functional Mock-up Interface - www.fmistandard.org

[5] HLA standard - IEEE standard for modeling and simulation (M\&S) high-level architec- ture (HLA) - framework and rules ieexplore.ieee.org/servlet/opac?punumber=7179.

[6] E. Lee, "Cyber physical systems: Design challenges," in Proc. of the 11th IEEE Int'l. Symposium on Object Oriented Real-Time Distributed Computing (ISORC'08), May 2008, pp. 363-369.

[7] T. Blochwitz, M. Otter, M. Arnold, C. Bausch, C. Clauß, H.Elmqvist, A. Junghanns, J. Mauss, M. Monteiro, T. Neidhold, D. Neumerkel, H. Olsson, J. V. Peetz, S. Wolf, "The Functional Mockup Interface for Tool independent Exchange of Simulation Models", in 8th International Modelica Conference, Dresden, 2011, pp. 20-22.

[8] Modelica Association: Modelica - A Unified Object-Oriented Language for Physical Systems Modeling. Language Specification, Version 3.2, March 24, 2010: www.modelica.org/documentas/ModelicaSpe c32.pdf

[9] Awais, M.U.; Palensky, P.; Elsheikh, A.; Widl, E.; Matthias, S., "The high level architecture RTI as a master to the functional mock-up interface components," Computing, Networking and Communications (ICNC), 2013 International Conference on, vol., no., pp.315,320, 28-31 Jan. 2013 doi: 10.1109/ICCNC.2013.6504102

[10] Portico RTI - www.porticoproject.org

[11] Sztipanovits, J., and Karsai, G. 1997. "Model-Integrated Computing", IEEE Computer, 30(110-112)

[12] de Laura, J., and Vangheluwe, H., 2002. "AToM3: A Tool for Multi-formalism and Meta-Modeling", Lecture Notes in Computer Science, 2306 (174-188).

[13] Tolvanen, J.P., and Lyytinen, K. 1993. "Flexible Method Adaptation in CASE. The Metamodeling Approach", Scandinavian Journal of Information Science, v5 n1 (7177).

[14] Cook, S., Jones, G., Kent, S., and Wills, A. 2007. "Domain-specific Development with Visual Studio DSL Tools", Addison-Wesley Professional

[15] The Eclipse Foundation - www.eclipse.org

[16] Modelon, Inc. - www.modelon.com

[17] JFMI: A Java wrapper for the Functional Mockup Interface www.ptolemy.eecs.berkeley.edu/java/jfmi

[18] OMNeT++ - www.omnetpp.org 
[19] DARPA Adaptive Vehicle Make Program www.darpa.mil/Our_Work/TTO/Programs/A daptive_Vehicle_Make_(AVM).aspx

[20] Jing Lin; Sedigh, S.; Miller, A., "Towards Integrated Simulation of Cyber-Physical Systems: A Case Study on Intelligent Water Distribution," Dependable, Autonomic and Secure Computing, 2009. DASC '09. Eighth IEEE International Conference on, vol., no., pp.690,695, 12-14 Dec. 2009. doi: 10.1109/DASC.2009.140.

[21] Palachi, E.; Cohen, C.; Takashi, S., "Simulation of cyber physical models using SysML and numerical solvers," Systems Conference (SysCon), 2013 IEEE International, vol., no., pp.671,675, 15-18 April 2013. doi: 10.1109/SysCon.2013.6549954.

[22] A. Al-Hammouri, V. Liberatore, H. AlOmari, Z. Al-Qudah, M. S. Branicky, and D. Agrawal, "A co-simulation platform for actuator networks," in Proc. of the 5th Int'l. Conference on Embedded Networked Sensor Systems (SenSys '07). New York, NY, USA: ACM, 2007, pp. 383-384.

[23] A. Bakshi, V. K. Prasanna, and A. Ledeczi. 2001, "MILAN: A Model Based Integrated Simulation Framework for Design of Embedded Systems," SIGPLAN Not. 36, 8 (August 2001), 82-93. DOI=10.1145/384196.384210.

[24] Agrawal, A.; Ledeczi, A., "Multigranular simulation of heterogeneous embedded systems," Engineering of Computer-Based Systems, 2003. Proceedings. 10th IEEE International Conference and Workshop on the , vol., no., pp.3,10, 7-10 April 2003. doi: 10.1109/ECBS.2003.1194776.

[25] J. Bastian, Ch. Clauß, S. Wolf, P. Schneider, "Master for Co-Simulation Using FMI," 8th International Modelica Conference. Dresden 2011.

[26] De Filippo, F.; Stork, A.; Schmedt, H.; Bruno, F, "A modular architecture for a driving simulator based on the FDMU approach," International Journal on Interactive Design and Manufacturing (IJIDeM) (2013): 1-12 , March 09, 2013.

[27] Lasnier, Gilles and Cardoso, Janette and Siron, Pierre and Pagetti, Claire and Derler, $\mathrm{Pa}$ tricia, "Distributed Simulation of Heterogeneous and Real-time Systems," In 17th IEEE/ACM International Symposium on Distributed Simulation and Real Time Appli- cations - IEEE/ACM DS-RT 2013, 30 October 2013 - 01 November 2013 (Delft, Netherlands).

[28] B. Wang and J. S. Baras, "HybridSim: A Modeling and Co-simulation Toolchain for Cyber-Physical Systems," Proceedings of 17th IEEE/ACM International Symposium on Distributed Simulation and Real Time Applications, pp. no. 33-40, Delft, Netherlands, October 30 - November 1, 2013.

[29] Elsheikh, A.; Awais, M.U.; Widl, E., Palensky, P., "Modelica-enabled rapid prototyping of cyber-physical energy systems via the functional mockup interface," Modeling and Simulation of Cyber-Physical Energy Systems (MSCPES), 2013 Workshop on , vol., no., pp.1,6, 20-20 May 2013. doi: 10.1109/MSCPES.2013.6623315.

[30] Awais, Muhammad Usman; Palensky, Peter; Mueller, Wolfgang; Widl, Edmund; Elsheikh, Atiyah, "Distributed hybrid simulation using the HLA and the Functional Mock-up Interface," Industrial Electronics Society, IECON 2013 - 39th Annual Conference of the IEEE, vol., no., pp.7564,7569, 10-13 Nov. 2013. doi: 10.1109/IECON.2013.6700393.

[31] Awais, Muhammad Usman, Wolfgang Mueller, Atiyah Elsheikh, Peter Palensky, and Edmund Widl, "Using the HLA for Distributed Continuous Simulations," In proceeding of: The 8th EUROSIM Congress on Modelling and Simulation. Sep. 2013.

[32] Karsai, G.; Sztipanovits, J.; Ledeczi, A.; Bapty, T., "Model-integrated development of embedded software," Proceedings of the IEEE , vol.91, no.1, pp.145,164, Jan 2003. doi: 10.1109/JPROC.2002.805824.

[33] Gabor Karsai , Janos Sztipanovits, "ModelIntegrated Development of Cyber-Physical Systems," Proceedings of the 6th IFIP WG 10.2 international workshop on Software Technologies for Embedded and Ubiquitous Systems, October 01-03, 2008, Anacarpi, Capri Island, Italy doi: 10.1007/978-3-54087785-1_5.

[34] D. Riley, E. Eyisi, J. Bai, X. Koutsoukos, Y. Xue, and J. Sztipanovits, "Networked control system wind tunnel (NCSWT): an evaluation tool for networked multi-agent systems," in Proceedings of the 4th International ICST Conference on Simulation Tools and Techniques (SIMUTools), 2011, pp. 9-18. 